\title{
Effect of maturation on muscle quality of the lower limb muscles in adolescent boys
}

\author{
Yuko Fukunaga, Yohei Takai", Takaya Yoshimoto, Eiji Fujita, Masayoshi Yamamoto and Hiroaki Kanehisa
}

\begin{abstract}
Background: The purpose of this study was to clarify the effect of maturation on the muscle quality of the lower limb muscles around puberty.

Methods: Subjects were 117 Japanese boys age 12 to 15 years. The maturity status was assessed by using a self-assessment of stage of pubic hair development based on the criteria of Tanner. On the basis of the criteria, subjects were divided into the prepubescent or pubescent group. Muscle thickness of knee extensors and plantar flexors were measured by a B-mode ultrasound. Muscle volume index (MV) was calculated from muscle thickness and limb length. Maximal voluntary isometric joint toques (TQ) of knee extension and ankle plantar flexion were measured using a myometer. Muscle quality was derived from dividing TQ by MV (TQ/MV).
\end{abstract}

Results: In both muscles, TQ-MV relationships were also similar between the prepubescent and pubescent groups, and there was no significant difference in TQ/MV between the two groups when chronological age was statistically adjusted.

Conclusion: The current results indicate that, for adolescent boys, the muscle quality of the lower limb muscles is not significantly influenced by maturation.

Keywords: Puberty, Maximal voluntary contraction, Muscle volume, Pubic hair, TQ-MV relationship

\section{Introduction}

It is well-known that, in adults, muscle size is a major determinant of force production capacity [1-6]. On the other hand, the earlier findings on the relationship between force-production capacity and muscle size during growth period differ among studies and among muscle groups tested [7-13]. One reason is that growth of both strength and muscle size is affected by chronological age and maturation [14]. Furthermore, methodological difference in determining muscle size [15] may also be a reason for the aforementioned discrepancy among the previous findings on strength-size relationships. The specific tension of a muscle is theoretically determined as muscle force relative to the physiological cross-sectional area (PCSA). To determine precisely the PCSA of a muscle in vivo is difficult because of the need to measure muscle volume, muscle fiber length, and fiber pennation angle [16], but it is difficult to measure these precisely in vivo. Some researcher have used the maximal voluntary joint toque

\footnotetext{
* Correspondence: y-takai@nifs-k.ac.jp

National Institute of Fitness and Sports in Kanoya, 1 Shiromizu, Kanoya, Kagoshima 8912393, Japan
}

(TQ) per muscle volume (TQ/MV) as an index of muscle force per PCSA [6], and this index is expressed as muscle quality $[17,18]$. Furthermore, Akagi et al. [4] have demonstrated that MV compared to muscle anatomical crosssectional area is appropriate for evaluating the strength-size relationship in elbow flexors. These aspects indicate that maturity-related difference in strength-size relationships should be examined by using the TQ-MV relationship.

Some studies have already examined the TQ-MV relationship and muscle quality in periods of growth $[12,13,19]$. However, the issue concerning the influence of maturation on muscle quality is still controversial. Pitcher et al. [19] demonstrated that $\mathrm{TQ} / \mathrm{MV}$ in the knee extensors was constant for 6 months in the period of preadolescence. On the other hand, the specific force of the gastrocnemius muscle is higher in early prepubescent boys than in adults [13]. Knee extensor muscle strength in boys is influenced not only by body size but also by testosterone level [20], which becomes an indicator of maturation. Serum testosterone level is positively related to maximal isokinetic knee extension in adolescent boys [21]. Taken together, 
muscle quality of the lower extremity muscles would be influenced by maturation.

The earlier findings cited above have been obtained by comparing prepubescent boys with adults. During adolescence, body size markedly changes with advancing chronological age and maturation, and its change accompanies with increases in muscle size and strength $[22,23]$. It has been shown that not only chronological age but also the magnitude of maturity influences the development of qualitative factors such as muscle strength, fiber composition, glycolytic and motor coordination $[20,21,24]$. This may complicate the interpretation of TQ-MV relationship in growth period. It should be necessary to determine the effect of maturation on muscle quality within a limited chronological age range in order to reduce the influence of chronological age on maturityrelated difference $[25,26]$. To the best of our knowledge, less information on the influence of maturation on the TQ-MV relationship around puberty is available from earlier studies. Force production capacity of the lower extremity muscles (for example, the knee extensors and ankle plantar flexors) is associated with sprint and jump performances $[12,26,27]$. To clarify the influence of maturation on muscle quality is feasible for coaches and physical educators involved in pediatric exercise. Hence, the purpose of this study is to clarify the effect of maturation on muscle quality in the lower extremity muscles around puberty for adolescent boys. Considering the earlier findings cited above, we hypothesized that the TQ-MV relationship in the lower limb muscles would differ between prepubescent and pubescent boys, and the muscle quality might be higher in pubescent than in prepubescent boys.

\section{Methods}

Subjects

One hundred and seventeen boys age 12 to 15 years participated in this study. Prior to the experiment, this study was approved by the Ethical Committee of the National Institute of Fitness and Sports in Kanoya and was consistent with their requirements for human experimentation. All subjects and their parents were informed of the purpose and procedures of this study and possible risks of the measurements beforehand. Written informed consent was obtained from each subject and parent.

\section{Experimental design}

Malina et al. [14] reported that Tanner stage I and II indicates criteria of preadolescence period. Age at peak height velocity, an index of puberty onset, is 13 years for Japanese boys [28-30]. We have also demonstrated that body height at peak height velocity in Japanese boys (approximately $154 \mathrm{~cm}$ ) corresponded to that between Tanner stage II and III [26]. On the basis of the maturity status, therefore, the subjects were allocated to the prepubescent group ( $n=47$, Tanner stage I to II) or the pubescent group $(\mathrm{n}=70$, Tanner stage III to V). All procedures were conducted according to our previous studies [25,26,31].

\section{Assessment of sexual maturation}

A self-assessment of stage of pubic hair $(\mathrm{PH})$ based on the criteria of Tanner [32], which was illustrated with black and white, was used to evaluate secondary sex characteristics. To reduce embarrassment, each subject went into a room by himself to complete the self-assessment anonymously [33]. Once completed, the self-assessment form was put into a box set in the room. The stage of $\mathrm{PH}$ consisted of five classes.

\section{Measurements of muscle thickness}

Muscle thickness of knee extensors (KE) and ankle plantar flexors $(\mathrm{PF})$ were measured with a B-mode ultrasonographic apparatus (Prosound2, Aloka, Tokyo, Japan) with a linear scanner. As described in the earlier study [34], the ultrasonographic images were obtained at $50 \%$ of femur length (the distance from the greater trochanter of the femur to the articular cleft between the femur and the tibial condyles) and the proximal 30\% of lower leg length (the distance from the tibial condyles and lateral malleolus). The muscle thickness (MT) was defined as the distance from adipose tissue-muscle and bone interface. The muscle volume indices (MV) of the knee extensors and ankle plantar flexors were calculated using the prediction equations derived from MT and limb length (L) reported by Miyatani et al. [35]:

MV in the knee extensors $\left(\mathrm{cm}^{3}\right)$ $=(\mathrm{MT}(\mathrm{cm}) \times 320.6)+(\mathrm{L}(\mathrm{cm}) \times 110.9)-4437.9$

MV in the ankle plantar flexors $\left(\mathrm{cm}^{3}\right)$

$$
=(\mathrm{MT}(\mathrm{cm}) \times 219.9)+(\mathrm{L}(\mathrm{cm}) \times 31.3)-1758.0,
$$

and muscle quality was expressed as TQ relative to MV (TQ/MV) [18] in each muscle.

\section{Measurements of maximal voluntary isometric joint torque}

Maximal voluntary isometric joint torque (MVC) in knee extension and ankle plantar flexion was measured using a specially designed myometer (TAKEI, Niigata, Japan). The right leg was measured for all subjects. In the KE measurement, the subjects sat on the machine with a 90-degree angle at hip and knee joints. The subject's hip was fixed by a non-elastic belt to prevent his hip from moving. Knee extension torque (KET) was calculated by multiplying the knee extension force by the lower leg length. In the planter flexion measurement, the subjects sat on the machine with knee extended. The ankle angle was 90 degrees. The subject's ankle was secured by a non-elastic 
belt to prevent from moving. Planter flexion torque (PFT) was obtained in the myometer. The subjects gradually exerted muscle force from rest to maximum in 3 to 4 seconds and then sustained this force at the maximum for approximately 2 seconds. Subjects performed at least two MVC trials with a 2-minute rest between trials. If the difference in the MVC torque between two trials was $>10 \%$, an additional MVC trial was performed. The highest value among the trials was adopted for analysis.

\section{Statistical Analysis}

Descriptive data are presented as means \pm SDs. To test comparison between groups, an unpaired $t$-test was used. A one-way analysis of variance (ANOVA) was conducted to compare the maturity-related differences in the measured variables. An analysis of covariance (ANCOVA) was tested to assess the maturity-related difference in $\mathrm{TQ} / \mathrm{MV}$ when adjusting chronological age as covariate, and a Bonferroni post hoc test was used for comparison between groups within same sex. Pearson's product-moment correlation coefficient $(r)$ was calculated to determine the relationship between TQ and MV in both muscles

Table 1 Physical characteristics of prepubescent and pubescent boys

\begin{tabular}{llll}
\hline & Prepubescent & Pubescent & Effect size $(r)$ \\
\hline Age, years & $13.3 \pm 0.5$ & $14.0 \pm 0.6^{*}$ & 0.49 \\
Height, cm & $152.7 \pm 8.5$ & $163.6 \pm 6.5^{*}$ & 0.59 \\
Weight, kg & $42.2 \pm 8.0$ & $51.7 \pm 7.2^{*}$ & 0.53 \\
BMI, kg/m ${ }^{2}$ & $18.0 \pm 1.8$ & $19.2 \pm 1.5^{*}$ & 0.36 \\
Percent body fat, \% & $13.6 \pm 5.2$ & $15.4 \pm 3.9^{*}$ & 0.20 \\
LBM, kg & $36.2 \pm 5.2$ & $43.5 \pm 4.7^{*}$ & 0.60 \\
Limb length, cm & & & \\
Femur & $36.7 \pm 2.9$ & $38.6 \pm 2.1^{*}$ & 0.10 \\
Lower leg & $36.1 \pm 2.5$ & $38.2 \pm 1.8^{*}$ & 0.45 \\
Muscle thickness, cm & & & \\
KE & $4.0 \pm 0.5$ & $4.6 \pm 0.5^{*}$ & 0.49 \\
PF & $5.8 \pm 0.5$ & $6.4 \pm 0.5^{*}$ & 0.53 \\
Muscle volume index, cm & & \\
KE & $930 \pm 390$ & $1318 \pm 310^{*}$ & 0.51 \\
PF & $649 \pm 152$ & $850 \pm 139^{*}$ & 0.55 \\
Maximal voluntary joint torque, Nm & & \\
KET & $119.5 \pm 36.7$ & $160.0 \pm 39.0^{*}$ & 0.47 \\
PFT & $78.6 \pm 26.5$ & $111.7 \pm 34.2^{*}$ & 0.46 \\
TQ/MV, Nm/cm ${ }^{3}$ & & & \\
KET/MV & $0.14 \pm 0.05$ & $0.13 \pm 0.03^{*}$ & 0.20 \\
PFT/MV & $0.12 \pm 0.03$ & $0.13 \pm 0.03$ & 0.15 \\
\hline
\end{tabular}

*indicates significant difference between prepubescent and pubescent groups. $\mathrm{BMI}$, body mass index; KET, knee extension torque; LBM, lean body mass; MV, muscle volume index; PFT, ankle plantar flexion torque; $\mathrm{QF}$, thigh anterior; $\mathrm{TQ}$, maximal voluntary isometric joint torque; TS, posterior of lower leg. for each group. We compared the slopes and y-intercepts of regression lines from the TQ-MV relationships in both muscles between groups, and tested whether the $\mathrm{y}$-intercept for each regression line differed from 0 . Effect size was classified as trivial $\left(r<0.1, \eta^{2}<0.01\right)$, small $(r=0.1$ to $0.3, \eta^{2}=0.01$ to 0.06 ), moderate ( $r=0.3$ to $0.5, \eta^{2}=0.06$ to 0.14$)$, and large $\left(r>0.5, \eta^{2}>0.14\right)$ [36]. Statistical significance was set at $P<0.05$. All statistical procedures were conducted by using statistical software (SPSS 22.0 for windows, IBM, Japan).

\section{Results}

The physical characteristics of the subjects are presented in Table 1. All measured variables except for KET/MV and PFT/MV were higher in the pubescent group than in the prepubescent group. TQ was significantly correlated with $\mathrm{MV}$ in both muscles $(r=0.47$ to $0.70, P<0.05$, Figure 1) with a moderate to large effect. The slopes and $y$-intercepts of the regression lines in the corresponding relationships did not significantly differ between the two groups. In both groups, the y-intercept of the regression line was significantly different from zero in the knee extensors, but not in the ankle plantar flexors. In the

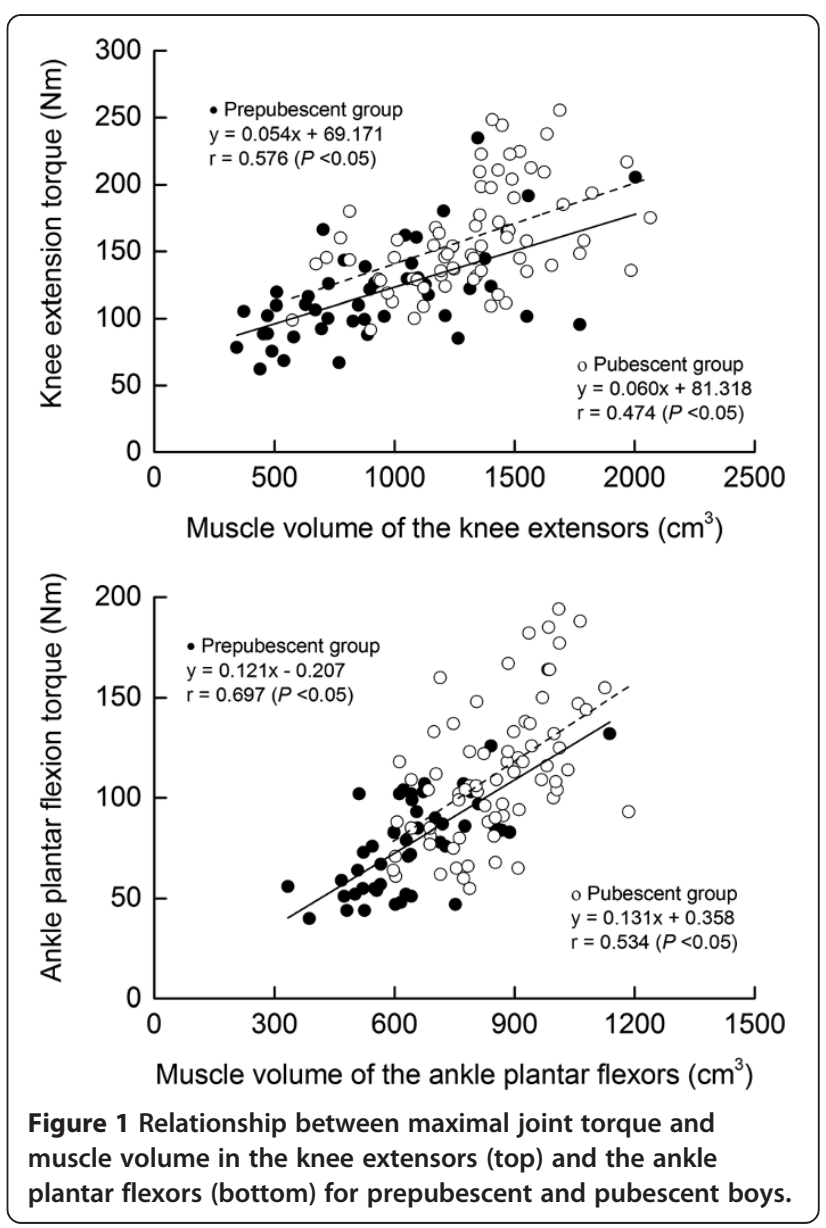


knee extensors and ankle plantar flexors, no significant difference in $\mathrm{TQ} / \mathrm{MV}$ was found between the two groups when chronological age was statistically adjusted as covariate.

The physical characteristics of each $\mathrm{PH}$ stage are shown in Table 2. In most of the measured variables, the significant differences were observed between $\mathrm{PH}$ I to II and $\mathrm{PH}$ III to $\mathrm{V}$ with a moderate and large effect size. There was no significant difference in KET/MV and PFT/MV among pubertal stages. As the result of ANCOVA, in which chronological age was adjusted as covariate, no significant difference in $\mathrm{TQ} / \mathrm{MV}$ was found in either muscle.

\section{Discussion}

The main finding obtained here was that isometric maximal joint torques relative to muscle volume in the knee extensor and ankle plantar flexor muscles were not different between the prepubescent and pubescent groups when chronological age was adjusted. This indicates that maturation has little influence on the muscle quality of lower extremity muscles in adolescent boys.

There were significant differences between the pubescent and prepubescent boys in all measured variables except for KET/MV and PFT/MV. The height in the prepubescent boys was small compared to that at peak height velocity of Japanese boys (approximately $154 \mathrm{~cm}$ ) [28-30]. During puberty, body size changes markedly with advancing chronological age and maturation, and its change accompanies an increase in muscle size and strength [23]. In this study, the subjects were sampled within a limited age range in order to reduce the confounding factor of chronological age. In general, more mature boys are taller and heavier than less mature boys. Thus, the current results reflect the characteristics of normal growth for adolescent boys.

Regardless of the prepubescent and pubescent groups, no significant maturity-related difference was found in the slopes and y-intercepts of the regression lines in the TQ-MV relationships in either muscle, indicating that the TQ-MV relationship in each muscle was similar between the prepubescent and pubescent boys. This is consistent with the earlier findings on the strength-size relationships in upper limb $[10,11]$ and the knee extensors $[12,19]$, but not with that in the gastrocnemius muscle [13]. The discrepancy in the result on the plantar flexors might be attributed to the difference in the subjects examined: prepubescent versus pubescent boys in this study and prepubescent boys versus adults in the earlier

Table 2 Maturity-related differences in anthropometry, body composition and maximal voluntary joint torque in adolescent boys

\begin{tabular}{|c|c|c|c|c|c|c|c|}
\hline & PH I & PH II & PH III & PH IV & PH V & Significance & Effect size $\left(\eta^{2}\right)$ \\
\hline Age, years & $13.0 \pm 0.4$ & $13.3 \pm 0.6$ & $13.7 \pm 0.6$ & $14.0 \pm 0.6$ & $14.1 \pm 0.6$ & $1<3$ to $5,2<4-5$ & 0.19 \\
\hline Height, cm & $146.6 \pm 8.1$ & $154.6 \pm 7.7$ & $159.7 \pm 7.1$ & $165.1 \pm 5.8$ & $165.4 \pm 5.3$ & $1<2$ to $5,2<4$ to $5,3<4$ & 0.20 \\
\hline Weight, kg & $38.8 \pm 6.4$ & $43.2 \pm 8.2$ & $47.4 \pm 7.0$ & $53.7 \pm 5.6$ & $53.4 \pm 7.7$ & $1<3$ to $5,2<4$ to $5,3<4$ & 0.13 \\
\hline $\mathrm{BMI}, \mathrm{kg} / \mathrm{m}^{2}$ & $18.0 \pm 1.6$ & $18.0 \pm 1.9$ & $18.5 \pm 1.3$ & $19.6 \pm 1.3$ & $19.4 \pm 1.7$ & $1<4,2<4$ to 5 & 0.03 \\
\hline Percent body fat, $\%$ & $13.8 \pm 3.3$ & $13.6 \pm 5.7$ & $14.1 \pm 3.4$ & $15.8 \pm 3.9$ & $16.3 \pm 4.2$ & & 0.01 \\
\hline LBM, kg & $33.3 \pm 4.3$ & $37.0 \pm 5.2$ & $40.6 \pm 4.8$ & $45.1 \pm 4.0$ & $44.4 \pm 4.4$ & $1,2<3$ to $5,3<4$ & 0.20 \\
\hline \multicolumn{8}{|l|}{ Limb length, $\mathrm{cm}$} \\
\hline Femur & $35.0 \pm 2.7$ & $37.3 \pm 2.7$ & $37.7 \pm 2.1$ & $39.3 \pm 2.0$ & $38.7 \pm 2.1$ & $1<3$ to $5,2<4$ & 0.05 \\
\hline Lower leg & $34.5 \pm 2.6$ & $36.6 \pm 2.3$ & $37.6 \pm 2.0$ & $38.5 \pm 1.6$ & $38.5 \pm 1.6$ & $1<2$ to $5,2<4-5$ & 0.08 \\
\hline \multicolumn{8}{|l|}{ Muscle thickness, $\mathrm{cm}$} \\
\hline KE & $4.1 \pm 0.5$ & $4.0 \pm 0.5$ & $4.3 \pm 0.5$ & $4.8 \pm 0.4$ & $4.6 \pm 0.4$ & $1,2<4$ to $5,3<4$ & 0.11 \\
\hline PF & $5.7 \pm 0.4$ & $5.8 \pm 0.5$ & $6.2 \pm 0.5$ & $6.6 \pm 0.4$ & $6.4 \pm 0.5$ & $1,2<3$ to $5,3<4$ & 0.12 \\
\hline \multicolumn{8}{|c|}{ Muscle volume index, $\mathrm{cm}^{3}$} \\
\hline KE & $748 \pm 380$ & $986 \pm 381$ & $1119 \pm 323$ & $1448 \pm 258$ & $1342 \pm 265$ & $1<3$ to $5,2<4$ to $5,3<4$ & 0.11 \\
\hline $\mathrm{PF}$ & $582 \pm 143$ & $670 \pm 150$ & $787 \pm 146$ & $899 \pm 115$ & $848 \pm 142$ & $1,2<3$ to $5,3<4$ & 0.15 \\
\hline \multicolumn{8}{|c|}{ Maximal voluntary joint torque, $\mathrm{Nm}$} \\
\hline KET & $103.8 \pm 29.7$ & $124.3 \pm 37.6$ & $147.7 \pm 36.3$ & $168.0 \pm 39.4$ & $161.6 \pm 39.9$ & $1<3$ to $5,2<4-5$ & 0.07 \\
\hline PFT & $59.9 \pm 16.9$ & $84.3 \pm 26.4$ & $107.5 \pm 39.3$ & $123.4 \pm 34.1$ & $100.4 \pm 23.8$ & $1<2$ to $5,2<4$ & 0.09 \\
\hline \multicolumn{8}{|l|}{$\mathrm{TQ} / \mathrm{MV}, \mathrm{Nm} / \mathrm{cm}^{3}$} \\
\hline KET/MV & $0.16 \pm 0.06$ & $0.14 \pm 0.05$ & $0.14 \pm 0.04$ & $0.12 \pm 0.03$ & $0.12 \pm 0.03$ & & 0.01 \\
\hline $\mathrm{PFT} / \mathrm{MV}$ & $0.11 \pm 0.03$ & $0.13 \pm 0.03$ & $0.14 \pm 0.04$ & $0.14 \pm 0.03$ & $0.12 \pm 0.03$ & & 0.01 \\
\hline
\end{tabular}

BMI, body mass index; KE, knee extensors; KET, knee extension torque; LBM, lean body mass; MV, muscle volume index; PF, ankle planter flexors; PFT, ankle plantar flexion torque; TQ, maximal voluntary isometric joint torque. 
study [13]. Furthermore, TQ/MV in both muscles was independent of the Tanner stage (Table 2). Tanner stage is associated with testosterone level [33]. Serum testosterone level is positively related to maximal isokinetic knee extension in adolescent boys [21]. In addition, voluntary activation level during maximal voluntary contraction [37,38], and proportion of fast type fiber [39] have been shown to be higher with increasing age. These findings will support the assumption that muscle quality might be higher in pubescent than in prepubescent boys, as hypothesized at the start of this study. However, this assumption is canceled by the current result that there was no significant difference between the prepubertal and pubertal groups in $\mathrm{TQ} / \mathrm{MV}$, with only a trivial or small effect size being observed.

We should comment on methodological issues with the estimation of muscle volume. The muscle volume was estimated using the prediction equation reported by Miyatani et al. [35], which has been derived from adult population. It has been shown that pennation angle, fascicle length relative to muscle length, and ratio of synergist muscle to total muscle volume is not different between prepubescent children and adults in the knee extensors [40] and ankle plantar flexors [13]. This implies that in an age span from childhood to adulthood, growth change in muscle volume is not associated with fascicle arrangement. In other words, the muscle thickness and limb length of children will be considered to be a scaled-down geometry of those in adults, and so the prediction equation derived from adult population can be used to estimate muscle volume for children. However, Midorikawa et al. [41] reported that while the muscle thickness-based prediction equation for adults are useful for estimating total and regional skeletal muscle mass for adolescents (Tanner stage $\geq \mathrm{II}$ ), this equation underestimates muscle mass in prepubescent children. If the previous finding can be applied to our data, the TQ/MV might be higher in the prepubertal group than in the pubertal group due to the underestimation of MV in the prepubertal group. However, the current result refutes this.

In the knee extensors, on the other hand, the yintercept of the regression line in the relationship between TQ and MV was significantly different from zero, but not for the ankle plantar flexors. This indicates that the higher $y$-intercept of the regression line from zero appears to result in the overestimation of the TQ/MV in the knee extensors in both groups. It is unknown whether this is due to the use of the prediction equation derived from an adult population. However, it should be noted that the significant difference in the y-intercept of the regression line from zero was found in both prepubertal and pubertal groups. In addition, the y-intercepts and slopes of regression lines in the corresponding relationships were similar between the two groups. These results imply that even if $\mathrm{TQ} / \mathrm{MV}$ for the knee extensors might be overestimated, its magnitude would be almost the same in both groups and have less influence on the current result that maturity-related difference was not found in KET/ MV. To generalize the current results, however, further investigation involving muscle volume measurements with more sophisticated techniques such as magnetic resonance imaging is needed.

\section{Conclusion}

The current results demonstrated that muscle quality, expressed as maximal joint torque relative to muscle volume, is not different between prepubescent and pubescent boys in the knee extensors and ankle plantar flexors. It suggests that maturation has little influence on the strength-size relationship in the lower extremity muscles around puberty. The current results were obtained from a cross-sectional survey. In a longitudinal survey, TQ/MV in the knee extensors is constant for the 6-month period of preadolescence [19]. However, to the best of our knowledge, less information on the longitudinal change in $\mathrm{TQ} / \mathrm{MV}$ in the period of adolescence is available from earlier findings. To clarify, this is important for pediatric exercise physiology, and further investigation based on longitudinal survey is needed.

\section{Abbreviations}

BMI: Body mass index; KE: Knee extensors; KET: Knee extension torque; L: Limb length; LBM: Lean body mass; MT: Muscle thickness; MV: Muscle volume;

MVC: Maximal voluntary isometric joint torque; PCSA: Physiological cross-sectional area; PF: Ankle plantar flexors; PFT: Ankle plantar flexion torque; PH: Stage of pubic hair; TQ: Maximal joint torque; \% fat: Percent body fat.

\section{Competing interests}

The authors declare that they have no competing interests.

\section{Authors' contributions}

YF carried out the anthropometric measurement, performed the statistical analysis and drafted the manuscript. YT conceived of the study, and participated in its design and coordination and helped to draft the manuscript. TY and EF carried out the strength measurement and helped to perform the statistical analysis. MY and HK supervised the survey, participated in the design of the study and performed the statistical analysis. All authors read and approved the final manuscript. All authors read and approved the final manuscript.

Received: 24 June 2014 Accepted: 3 September 2014 Published: 19 September 2014

\section{References}

1. Ikai $M$, Fukunaga T: Calculation of muscle strength per unit cross-sectional area of human muscle by means of ultrasonic measurement. Int $Z$ Angew Physiol 1968, 26:26-32.

2. Klein CS, Rice CL, Marsh GD: Normalized force, activation, and coactivation in the arm muscles of young and old men. J Appl Physiol (1985) 2001, 91:1341-1349.

3. Candow DG, Chilibeck PD: Differences in size, strength, and power of upper and lower body muscle groups in young and older men. J Gerontol A Biol Sci Med Sci 2005, 60:148-156.

4. Akagi R, Takai Y, Ohta M, Kanehisa H, Kawakami Y, Fukunaga T: Muscle volume compared to cross-sectional area is more appropriate for evaluating muscle strength in young and elderly individuals. Age Ageing 2009, 38:564-569. 
5. Akagi R, Takai $Y$, Ohta M, Kanehisa H, Fukunaga T, Kawakami Y: Size-strength relationships of the elbow flexors and extensors are not affected by age or gender. Eur J Sport Sci 2011, 11:277-282.

6. Fukunaga T, Miyatani M, Tachi M, Kouzaki M, Kawakami Y, Kanehisa H: Muscle volume is a major determinant of joint torque in humans. Acta Physiol Scand 2001, 172:249-255.

7. Kanehisa H, Ikegawa S, Tsunoda N, Fukunaga T: Strength and crosssectional areas of reciprocal muscle groups in the upper arm and thigh during adolescence. Int J Sports Med 1995, 16:54-60.

8. Kanehisa $\mathrm{H}$, Yata $\mathrm{H}$, Ikegawa $\mathrm{S}$, Fukunaga $\mathrm{T}$ : A cross-sectional study of the size and strength of the lower leg muscles during growth. Eur J App/ Physiol Occup Physiol 1995, 72:150-156.

9. Neu CM, Rauch F, Rittweger J, Manz F, Schoenau E: Influence of puberty on muscle development at the forearm. Am J Physiol Endocrinol Metab 2002, 283:E103-E107.

10. Wood LE, Dixon S, Grant C, Armstrong N: Elbow flexion and extension strength relative to body or muscle size in children. Med Sci Sports Exerc 2004, 36:1977-1984.

11. Tonson A, Ratel S, Le Fur Y, Cozzone P, Bendahan D: Effect of maturation on the relationship between muscle size and force production. Med SCi Sports Exerc 2008, 40:918-925.

12. O'Brien TD, Reeves ND, Baltzopoulos V, Jones DA, Maganaris CN: Strong relationships exist between muscle volume, joint power and whole-body external mechanical power in adults and children. Exp Physiol 2009, 94:731-738

13. Morse Cl, Tolfrey K, Thom JM, Vassilopoulos V, Maganaris CN, Narici MV: Gastrocnemius muscle specific force in boys and men. J Appl Physiol (1985) 2008, 104:469-474.

14. Malina RM, Bouchard C, Bar-Or O: Growth, Maturation, and Physical Activity. Champaign, IL: Human Kinetics; 2004.

15. Bouchant A, Martin V, Maffiuletti NA, Ratel S: Can muscle size fully account for strength differences between children and adults? J Appl Physiol (1985) 2011, 110:1748-1749.

16. Kawakami Y, Nakazawa K, Fujimoto T, Nozaki D, Miyashita M, Fukunaga T: Specific tension of elbow flexor and extensor muscles based on magnetic resonance imaging. Eur J Appl Physiol Occup Physiol 1994, 68:139-147.

17. Dutta C, Hadley EC, Lexell J: Sarcopenia and physical performance in old age: overview. Muscle Nerve 1997, 5:S5-S9.

18. Lynch NA, Metter EJ, Lindle RS, Fozard JL, Tobin JD, Roy TA, Fleg JL, Hurley BF: Muscle quality. I. Age-associated differences between arm and leg muscle groups. J Appl Physiol (1985) 1999, 86:188-194.

19. Pitcher CA, Elliott CM, Williams SA, Licari MK, Kuenzel A, Shipman PJ, Valentine JP, Reid SL: Childhood muscle morphology and strength alterations over six months of growth. Muscle Nerve 2012, 46:360-366.

20. Round JM, Jones DA, Honour JW, Nevill AM: Hormonal factors in the development of differences in strength between boys and girls during adolescence: a longitudinal study. Ann Hum Biol 1999, 26:49-62.

21. Ramos E, Frontera WR, Llopart A, Feliciano D: Muscle strength and hormonal levels in adolescents: gender related differences. Int J Sports Med 1998, 19:526-531.

22. Asmussen $\mathrm{E}$, HeebOOll-Nielsen $\mathrm{K}$ : A dimensional analysis of physical performance and growth in boys. J Appl Physiol 1955, 7:593-603.

23. Parker DF, Round JM, Sacco P, Jones DA: A cross-sectional survey of upper and lower limb strength in boys and girls during childhood and adolescence. Ann Hum Biol 1990, 17:199-211.

24. Martin RJ, Dore E, Hautier CA, Van Praagh E, Bedu M: Short-term peak power changes in adolescents of similar anthropometric characteristics. Med Sci Sports Exerc 2003, 35:1436-1440.

25. Fukunaga $Y$, Takai $Y$, Yoshimoto T, Fujita E, Yamamoto M, Kanehisa H: Influence of maturation on anthropometry and body composition in Japanese junior high school students. J Physiol Anthropol 2013, 32:5.

26. Yoshimoto T, Takai Y, Fukunaga Y, Fujita E, Kanehisa H, Yamamoto M: Effect of maturation on sprint and jump performances in adolescent boys. Gazz Med Ital 2014, 173:265-272.

27. Yoshimoto T, Takai Y, Fujita E, Fukunaga Y, Kintaka H, Nishizono H, Kanehisa $H_{\text {, }}$ Yamamoto M: Influence of the torque generating capacity of the lower extremity muscles on the running and jump performance in primary and junior high school boys. JJPFSM 2012, 61:79-88 [in Japanese].
28. Kobayashi K, Kitamura K, Miura M, Sodeyama H, Murase Y, Miyashita M, Matsui $\mathrm{H}$ : Aerobic power as related to body growth and training in Japanese boys: a longitudinal study. J Appl Physiol 1978, 44:666-672.

29. Suwa S, Tachibana K, Maesaka H, Tanaka T, Yokoya S: Longitudinal standards for height and height velocity for Japanese children from birth to maturity. Clin Pediatric Endocrin 1992, 1:5-13.

30. Nariyama K, Hauspie RC, Mino T: Longitudinal growth study of male Japanese junior high school athletes. Am J Hum Biol 2001, 13:356-364.

31. Takai Y, Fukunaga Y, Fujita E, Mori H, Yoshimoto T, Yamamoto M, Kanehisa $\mathrm{H}$ : Effects of body mass-based squat training in adolescent boys. J Sports Sci Med 2013, 12:60-65.

32. Tanner JM: Growth at Adolescence. Oxford: Blackwell Scientific Publications; 1962.

33. Gurd B, Klentrou P: Physical and pubertal development in young male gymnasts. J Appl Physiol 2003, 95:1011-1015.

34. Abe T, Kondo M, Kawakami Y, Fukunaga T: Prediction equations for body-composition of Japanese adults by B-mode ultrasound. Am J Hum Biol 1994, 6:161-170

35. Miyatani $M$, Kanehisa $H$, Ito $M$, Kawakami $Y$, Fukunaga $T$ : The accuracy of volume estimates using ultrasound muscle thickness measurements in different muscle groups. Eur J Appl Physiol 2004, 91:264-272.

36. Cohen J: Statistical Power Analysis for the Behavioral Sciences. Hillsdale, NJ. Lawrence Erlbaum; 1988

37. Belanger AY, McComas AJ: Contractile properties of human skeletal muscle in childhood and adolescence. Eur J Appl Physiol Occup Physiol 1989, 58:563-567.

38. O'Brien TD, Reeves ND, Baltzopoulos V, Jones DA, Maganaris CN: In vivo measurements of muscle specific tension in adults and children. Exp Physiol 2010, 95:202-210.

39. Lexell J, Sjostrom M, Nordlund AS, Taylor CC: Growth and development of human muscle: a quantitative morphological study of whole vastus lateralis from childhood to adult age. Muscle Nerve 1992, 15:404-409.

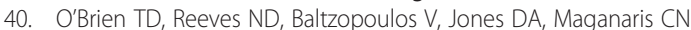
Muscle-tendon structure and dimensions in adults and children. J Anat 2010, 216:631-642

41. Midorikawa T, Sanada K, Yoshitomi A, Abe T: Is the use of ultrasound-derived prediction equations for adults useful for estimating total and regional skeletal muscle mass in Japanese children? Br J Nutr 2009, 101:72-78.

doi:10.1186/1880-6805-33-30

Cite this article as: Fukunaga et al.: Effect of maturation on muscle quality of the lower limb muscles in adolescent boys. Journal of Physiological Anthropology 2014 33:30.

\section{Submit your next manuscript to BioMed Central and take full advantage of:}

- Convenient online submission

- Thorough peer review

- No space constraints or color figure charges

- Immediate publication on acceptance

- Inclusion in PubMed, CAS, Scopus and Google Scholar

- Research which is freely available for redistribution 